\title{
Diversidad de la familia Orchidaceae en los bosques montanos de San Ignacio (Cajamarca, Perú)
}

\author{
Diversity of Orchidaceae family in the montane forest in San Ignacio \\ (Cajamarca, Peru)
}

\author{
Gloria Calatayud*
}

Publicación: 08/12/2005

\section{Resumen}

La insuficiente información sobre la flora, específicamente sobre la familia Orchidaceae y la inminente desaparición de los bosques en la Vertiente Oriental del río Chinchipe (San Ignacio, Cajamarca), fueron las razones suficientes que motivaron a realizar la presente investigación. La evaluación se realizó en cuatro localidades de la provincia San Ignacio (Cajamarca), durante el año 2000 , con 27 cuadrantes de $500 \mathrm{~m}^{2}$, distribuidos cada $100 \mathrm{~m}$ de altitud, desde los 800 hasta los 2700 $\mathrm{m}$, el método de muestreo fue aleatorio. Según el índice de diversidad de Shannon-Wiener, la zona de estudio posee un alto grado de diversidad (entre $H^{\prime}=5,93$ en Selva Andina y H'= 4,02 en Camaná), gracias al buen estado de conservación de los bosques y a la topografía de la zona. Las localidades de Camaná-Crucero $(173,55)$ son menos disímiles por presentar hábitats similares. Selva Andina $(969,619)$ es la localidad más disímil con el grupo Crucero-Camaná-Nuevo Mundo, la cual a los 2700 $\mathrm{m}$ presenta un alto endemismo. Para las localidades evaluadas se reportan 205 especies distribuidas en 58 géneros, los cuales son reportes nuevos para la zona, 15 son registros nuevos para Perú, ocho de éstas dejan de ser endémicas para Ecuador. Se reporta por primera vez para Perú el género Chrysocycnis, y dos especies nuevas para la ciencia Sarcoglottis sp. y Maxillaria sp.

Palabras clave: Diversidad, Endemísmo, hábitat, conservación, deforestación, Perú

\section{Abstract}

The insufficient information on the flora, specifically on the Orchidaceae family and the imminent destruction of the forests in the Eastern slope of the Chinchipe river (San Ignacio, Cajamarca), were the reasons that motivated to make the present investigation. The evaluation was carried out in four localities of the county San Ignacio (Cajamarca), during year 2000. Sampling was random with 27 quadrants an altitudinal range of $100 \mathrm{~m}$ and comprising an area of $500 \mathrm{~m}^{2}$ each. Plots were distributed between 800 and $2700 \mathrm{~m}$ above sea level. According to the Shannon-Wiener Diversity Index diversity is high in the study area (> grade: Loc. Selva Andina: $H^{\prime}=5,93$ and <grade: Loc. Camaná: $H^{\prime}=4,02$ ) and this is likely due to the currently good state of conservation of these forests and to the varied topography of the area. The localities of Camaná-Crucero $(173,55)$ have similar indices because they represent similar habitat types. The locality of Selva Andina (969619) shows high altitude endemism (the area reaches elevations of $2700 \mathrm{~m}$ ) and is thus dissimilar to the group CruceroCamaná-Nuevo Mundo. In total 205 species distributed in 58 genera are reported. 15 are reported as new records for Peru, eight of these were previously considered as Ecuadorian endemics. The genus Chrysocycnis is reported from Peru for the first time. Two species new to science are reported from the genera Sarcoglottis and Maxillaria.

Keywords: Diversity, Endemisms, Habitat, Conservation, Deforestation.

\section{Introducción}

En los últimos 100 años los bosques montanos de la provincia de San Ignacio (Cajamarca) han ido desapareciendo a paso acelerado debido a la extracción de madera y

\footnotetext{
*Jardín Botánico de Missouri-Perú

Urb. Ttio J 2-5, Pje. Bariloche, Huanchac-Cusco
}

la actividad ganadera que a su vez constituyen las principales actividades económicas de la zona y que convierten al bosque en pastizales de baja calidad o plantaciones de café. La mayor parte de este impacto ocurre a lo largo y dentro de las zonas cercanas a carreteras y pequeñas localidades o caseríos. 
En la zona de estudio aún existen áreas de bosque prístino y otras con poco disturbio antrópico, excepto la localidad de Crucero que apenas presenta algunos parches de bosque disturbado, con una diversidad biológica singular. La alta precipitación, la geología y topografía tan compleja contribuyen a crear y mantener ésta diversidad. Dichas tierras no son apropiadas para la agricultura, debido a lo somero de sus suelos, laderas con pendientes pronunciadas y lluvias torrentosas.

Como en cualquier parte, aquí en San Ignacio también la palabra «orquídea» está asociada con adjetivos de exótico, raro e inagotable, y en la mayoría de los casos son extraídas y comercializadas directamente del campo, lo cual reduce significativamente el número de éstas. Entonces es necesario establecer pautas muy claras y precisas acerca de su conservación o manejo sostenible, puesto que constituyen también patrimonio de nuestra nación. Es así que su investigación minuciosa y profunda reviste cada vez mayor importancia.

Uno de los objetivos del presente estudio fue evaluar la diversidad genérica y específica de la familia Orchidaceae, su similitud en las localidades en estudio, establecer los diferentes tipos de hábitats y determinar su distribución a través de la gradiente altitudinal.

En la vertiente oriental del río Chinchipe, muchos de los datos en cuanto a flora y fauna se desconocen y no se han realizado estudios específicos sobre esta familia; a ello se debe sumar la destrucción de los bosques por la extracción de madera y ganadería, razones suficientes que motivan a realizar la presente investigación.

\section{Material y métodos}

Se consideró materiales de colección y toma de datos, de prensado y secado, de descripción y de consulta: Biblioteca especializada del Jardín Botánico de Missouri con sede en la ciudad de Jaén, Herbario San Marcos (USM) del Museo de Historia Natural de la Universidad Nacional Mayor de San Marcos,
Biblioteca y Herbario del Missouri Botanical Garden (Missouri, St. Louis).

El área de estudio está ubicada en el departamento de Cajamarca, provincia de San Ignacio, distrito de San José de Lourdes (localidades de muestreo: Selva Andina, Crucero, Camaná) y distrito de Huarango (localidad de muestreo: Nuevo Mundo), entre los $04^{\circ} 18^{\prime}$ y $05^{\circ} 28^{\prime} \mathrm{S}$ y los $78^{\circ} 57^{\prime}$ y $79^{\circ} 23^{\prime} \mathrm{O}$; desde los 800 a los $2730 \mathrm{~m}$ de altitud.

La evaluación de campo se realizó durante los meses de julio a octubre y febrero-marzo de los años 2000 y 2001, con salidas mensuales de 15 días por mes en el mejor de los casos, abarcando así las épocas de estío y de lluvias.

El método de muestreo fue al azar, simple o aleatorio; el tamaño de la muestra se estableció sobre la base del área mínima de muestreo de 0,1 ha (Palomino, 1999) para bosques montanos, los cuales presentan caracteres geológico, topográfico, climático y florístico similares.

Las unidades muestrales fueron cuadrantes de $50 \times 10 \mathrm{~m}^{2}$ cada $100 \mathrm{~m}$ de altitud desde los $800 \mathrm{~m}$ sobre el nivel del mar (localidad de Crucero) hasta los $2730 \mathrm{~m}$ (localidad de Selva Andina, Cerro Picorana). También se colectó fuera del área de los cuadrantes, sin embargo estas muestras no fueron incluidas en el tratamiento estadístico pero sí en la lista de especies y descripción.

El total de unidades muestrales sumó 27. Los cuadrantes fueron trazados tomando como referencia trochas y carreteras, hacia las localidades y desde las localidades, así como también dentro del bosque tanto primario como secundario.

Para facilitar la determinación de las muestras se preservó en frascos de vidrio o plástico 1, 2 ó 3 flores, en solución: glicerina 5\%, agua destilada $25 \%$ y alcohol de $70 \%$.

El proceso de herborización se llevó a cabo en las oficinas del Jardín Botánico de Missouri (Cajamarca, prov. Jaén) el año 2000. Los especímenes se encuentran depositados en los 
herbarios de CUZ y USM (dispuestos según el número de duplicados existentes, los cuales van de acuerdo con el número de individuos encontrados en el área de trabajo). La determinación de las muestras botánicas fueron realizadas por la autora, en el Herbario San Marcos (USM) del Museo de Historia Natural de la Universidad Nacional Mayor de San Marcos y el Herbario y Biblioteca del Missouri Botanical Garden (St. Louis, Missouri, EE.UU.), mediante consulta de bibliografía especializada (claves de identificación botánica, patrones de descripción de especie, etc.), con el respaldo y colaboración del Ing. David Bennet Jr. (Biólogo), Ricardo Fernández (USM), Dr. Erick Chrystenson, Dr. Carl Luer (MO), Dr. Stig Dalstrom (SEL) y Dr. Hágsater (AMO).

Los datos de campo para el estudio de diversidad se trabajaron con los índices de Shannon-Wiener (Franco et al., 1995) e índice de Simpson (Krebs, 1989). Ya que la descripción de una comunidad nos lleva necesariamente a la comparación con otra o con ella misma en distintos tiempos, se ha realizado la medición de la disimilitud (Franco et al., 1995); para este análisis entre los cuadrantes evaluados se eligió el análisis cuantitativo de distancia euclidiana.

Basándonos en una matriz de disimilitud se realizó un análisis de agrupamiento entre parcelas, el que representa la secuencia de fusiones en un esquema dendrítico. La técnica utilizada es el de ligamento simple, se junta de a pares las muestras cuyos índices de similitud son mayores (Matteucci \& Colma, 1982).

\section{Resultados}

De acuerdo con la Tabla 1 la mayoría de los géneros presentes en la zona de estudio, por su afinidad geográfica (Mabberley, 1997), tienen origen neotropical lo cual nos indica que el origen y distribución de la familia Orchidaceae, en la provincia de San Ignacio es relativamente reciente en el tiempo.

En las cuatro localidades evaluadas de la provincia de San Ignacio (Cajamarca) se re-
Tabla 1. Afinidad geográfica de los géneros reportados para las cuatro localidades en la zona de estudio.

\begin{tabular}{ll}
\hline Zona fitogeográfica & Número de géneros \\
\hline América & 1 \\
América Tropical & 33 \\
Andes Tropicales & 1 \\
América Tropical, Norteamérica & 2 \\
Andes & 4 \\
Sudamérica & 1 \\
Sudamérica Tropical & 1 \\
Trópical y Cálida & 1 \\
América Tropical Cálida & 2 \\
\hline
\end{tabular}

gistró un total de 205 especies para 58 géneros, la localidad que presenta el mayor número de especies y géneros es Selva Andina con 98 especies distribuidas en 30 géneros, seguida de la localidad de Nuevo Mundo que muestra 50 especies distribuidas en 19 géneros; la localidad de Crucero presenta 47 especies para 26 géneros y finalmente la localidad de Camaná que alberga 38 especies distribuidas en 18 géneros. La Tabla 2 resume la diversidad de especies de la familia Orchidaceae para el área de estudio.

El índice de diversidad de Simpson (1-D), nos indica que la diversidad en el área de estudio es alta. El índice de diversidad de Shannon-Wiener $\left(\mathrm{H}^{\prime}\right)$ nos muestra que la localidad de Selva Andina presenta la mayor diversidad $\left(\mathrm{H}^{\prime}=5,93\right)$, seguida de la localidad de Nuevo Mundo $\left(\mathrm{H}^{\prime}=4,87\right)$, localidad de Crucero $\left(\mathrm{H}^{\prime}=4,81\right)$ y finalmente la localidad de Camaná $\left(\mathrm{H}^{\prime}=4,02\right)$ (Tabla 2).

Según los resultados del análisis del índice de diversidad de Shannon-Wiener, la localidad de Selva Andina presenta la mayor diversidad $\left(\mathrm{H}^{\prime}=5,93\right)$ y un valor de equitatividad alto $\left(\mathrm{J}^{\prime}=0,794\right)$ respecto a las tres localidades restantes (Tabla 2), lo cual nos indica que los individuos están bien distribuidos en los cuadrantes evaluados de esta localidad y como consecuencia de ello se da la alta diversidad de especies que presenta, influenciado por la diferencia altitudinal que va desde los 1800 hasta $2700 \mathrm{~m}$ y que está dentro de la zona de vida bosque húmedo Montano Bajo Tropical (bh-MBT) con 
Tabla 2. Diversidad de las localidades de Crucero, Camaná, Selva Andina y Nuevo Mundo. H'= indice de diversidad de Shannon-Wiener, 1-D= índice de diversidad de Simpson.

\begin{tabular}{lccccccc}
\hline Localidad & $\mathbf{N}^{\mathbf{0}}$ especies & $\mathbf{N}^{\mathbf{0}}$ individuos & $\mathbf{H}^{\prime}$ & $\mathbf{H}^{\prime}$ máxima & Equitatividad & 1-D & recíproco D \\
\hline Crucero & 47 & 596 & 4,81 & 7,47 & 0,643 & 0,955 & 22,3 \\
Camaná & 38 & 399 & 4,02 & 7,47 & 0,538 & 0,897 & 9,69 \\
Selva Alta & 98 & 1650 & 5,93 & 7,47 & 0,794 & 0,979 & 4,50 \\
Nuevo Mundo & 50 & 616 & 4,87 & 7,47 & 0,652 & 0,953 & 21,3 \\
\hline Total & 233 & 3261 & 6,82 & 7,47 & 0,913 & 0,988 & 84,8 \\
\hline
\end{tabular}

características fisiográficas y climáticas aptas para la alta diversidad de la familia en estudio con presencia de bosques montanos continuos y fragmentados, donde el mayor número de especies está ubicado a los 1900 y $2000 \mathrm{~m}$.

La localidad de Nuevo Mundo presenta una diversidad de $\left(\mathrm{H}^{\prime}=4,87\right)$ y una equitatividad igualmente mayor respecto a las dos localidades restantes (Tabla 2); así, este alto valor nos indicará que las especies e individuos están bien distribuidos y no se presentan por aglomerados. La evaluación se realizó en bosques primarios fragmentados en altitudes de 1200 a $1600 \mathrm{~m}$. En tercer lugar se ubica la localidad de Crucero, con una diversidad de $\mathrm{H}^{\prime}=4,81$ (Tabla 2), con un rango altitudinal que va desde los 800 a $1300 \mathrm{~m}$. Aquí los cuadrantes fueron evaluados en bosques secundarios y parches de bosques primarios muy disturbados donde la mayor parte de las especies reportadas tienen hospederos restringidos a las especies Manilkara bidentata, Hura crepitans y Myrsine olygophylla. De tal manera que se puede afirmar que en esta localidad se han formado interesantes microclimas y microhábitats a manera de refugios, los cuales otorgan características apropiadas para la presencia de las orquídeas a pesar del rango altitudinal y del clima cálido, el cual es más acentuado en las épocas de estío, con temperaturas que superan $\operatorname{los} 30^{\circ} \mathrm{C}$. Las medidas de conservación de los restos de bosque en esta localidad son urgentes.

La localidad de Camaná presenta la menor diversidad $\mathrm{H}^{\prime}=4,02$ (Tabla 2); el rango altitudinal de evaluación fue de 1500 a 2000 $\mathrm{m}$, con 38 especies para 18 géneros, ubicada en la zona de vida de bh-MBT, en bosques primarios fragmentados y parches de bosque aislados por los cultivos de café.

De acuerdo con el análisis del Tabla 3, se tiene como resultado que hay menor disimilitud entre las localidades de Camaná y Crucero $(d=173,55)$ y mayor disimilitud $(\mathrm{d}=269,929)$ entre la localidad de Selva Andina con el grupo Nuevo Mundo, Camaná, Crucero.

El valor más bajo de disimilitud $(173,55)$ está entre las localidades de Camaná y Crucero (Tabla 3, Fig. 1). Tenemos así que de 29 géneros presentes en ambas localidades 12 son comunes $(41,37 \%)$. Otras de las razones por la cual estas dos localidades tienen el valor más bajo de disimilitud sería por las características de hábitats similares; además, ambas localidades presentan parches de bosques aislados, los cuales actúan como refugio de orquídeas (especialmente en la localidad de Crucero) y se encuentran rodeados de monocultivos de café y pasturas.

La localidad con el grado más alto de disimilitud $(269,929)$ es Selva Andina (Tabla 3, Fig. 1). Así, de los 51 géneros presentes para ambos grupos (Selva Andina y Crucero-CamanáNuevo Mundo) 14 están presentes sólo en Selva Andina (27,45\%). Esta alta disimilitud se debe a que existe una clara dependencia respecto al hábitat, pues las localidades de Crucero, Camaná y Nuevo Mundo presentan en gran parte bosques primarios fragmentados y bosques secundarios, lo que no sucede en Selva Andina donde aún hay presencia de bosques primarios continuos y fragmentados. 
Tabla 3. Matriz de Distancia euclidiana entre las localidades de estudio.

\begin{tabular}{lcccc}
\hline & Crucero Camaná & $\begin{array}{c}\text { Selva } \\
\text { Andina }\end{array}$ & $\begin{array}{c}\text { Nuevo } \\
\text { Mundo }\end{array}$ \\
\hline Crucero & 0 & 173,55 & 271,88 & 186,49 \\
Camaná & 173,55 & 0 & 249,68 & 187,43 \\
Selva Andina & 271,88 & 249,68 & 0 & 269,93 \\
Nuevo Mundo & 186,49 & 187,43 & 269,93 & 0 \\
\hline
\end{tabular}

Respecto a la disimilitud entre cuadrantes, el valor más bajo $(1,41)$ se presenta entre los cuadrantes III, XI y XXIV (Figura 2). Esta baja disimilitud también se debe a que los cuadrantes XI y XXIV fueron evaluados en bosques primarios fragmentados donde se realizó la tala de grandes áreas y en el caso del cuadrante III, ésta estuvo ubicada en tierras de cultivo abandonado. Como consecuencia de dicho impacto se ve claramente que la composición florística es menor y, por lo tanto, los hospederos y calidad de suelos serán bajos, lo que ocasiona que el número de especies sea menor, presentando sólo 13 especies para el grupo de cuadrantes III, XI y XXIV.

El valor de alta disimilitud $(126,09)$ se observa entre el cuadrante XXII y el resto de cuadrantes (Fig. 2); esto nos indicaria que ambos grupos no son similares y poseen características propias de cada lugar; así vemos que el cuadrante XXII está ubicado en el límite altitudinal de la evaluación (2700 m), donde el bosque es enano con árboles hasta $5 \mathrm{~m}$ de alto, el relieve plano abierto y donde se aprecia una vegetación predominantemente herbácea con alto grado de endemismo (Vásquez, 1999). Este tipo de hábitat tan característico no se observa en todo el resto de cuadrantes, por lo tanto las características climáticas son bastante diferentes y como consecuencia la composición florística específica tanto arbórea como herbácea es muy diferente al resto del grupo de cuadrantes. Las especies presentes sólo en el cuadrante XXII son: Elleanthus aurantiacus (Lindl) Rchb., Epidendrum macrostachyum Lindl., Epidendrum sp b,
Epidendrum sp I, Pleurothallis siphonanta Luer y las especies compartidas (y sólo con una cuadrante: XX) son: Octomeria bicallosa Luer, Epidendrum sp c y Pleurothallis cassidis Luer. De estas especies $O$. bicallosa es endémica de la región norte de Perú y sur de Ecuador, lo cual podría confirmar el alto endemismo del cerro Picorana (cuadrante XXII).

Otro valor alto de disimilitud $(112,955)$ está dado por el cuadrante $\mathrm{V}$ y el resto de cuadrantes (Fig. 2). Ello se debe a que el cuadrante $\mathrm{V}$, ubicado a $1200 \mathrm{~m}$ de altitud, también presenta características ambientales diferentes al grupo de cuadrantes mencionados (incluso la diferencia es clara entre los cuadrantes de la misma localidad); así, el cuadrante V fue evaluado en un bosque secundario el cual actúa como un refugio para las orquídeas ya que presenta características diferentes al resto de cuadrantes de su misma localidad. Como consecuencia, la presencia de especies de orquídeas es restringida para este cuadrante: Bletia purpurea (Lamark) DC, Cyclopogon sp, Cyrtopodium punctatum (L.) Lindley, Dryadella minúscula Luer \& Escobar, Isochilus linearis (Jacquin) R.Brown, Maxillaria ochracea Reichb.f.\& Garay, Maxillaria scorpioidea Kraenzlin,Mormolyca peruviana C.Schweinfurth, Oncidium sp d, Phragmipedium boissierinum (Rchb.f.) Rolfe, Pleurothallis picta Lindl., Pleurothallis revoluta (R.\& P) Garay, pleurothallis tripterantha Reichembach f., Pleurothallis xanthoclora Reichembach f., Prostechea aemula (Lindley) W.E.Higgins, Sarcoglottis sp, Psilochilus $s p$ y Stenoptera $s p$, como exclusivas para el transecto V. De igual forma los géneros exclusivos para este cuadrante son: Cyrtopodium, Dryadella, Isochilus, Psilochilus, Sarcoglottis y Stenoptera. En resumen se tienen cuatro grupos notorios:

$1^{\circ}$ Cuadrante XXII y el grupo I, II, III..., XXVII.

$2^{\circ}$ Cuadrante $\mathrm{V}$ y el grupo I, II, III..., XXVII (excepto XXII).

$3^{\circ}$ Cuadrante XIII y el grupo I, II, III, IV, VI, VII, VIII, IX, XI, XVI; XVII; XVIII, XIX; XX, XXIV, XXV y XXVII. 
De acuerdo con estos resultados podemos afirmar que existe una clara dependencia respecto al hábitat, ya que todos los cuadrantes fueron evaluados en bosque primario fragmentado, bosque primario continuo y bosque secundario pero con características particulares de hábitat, relieve, diversidad florística, humedad, rango altitudinal y estado de conservación.

El alto porcentaje de especies epífitas se debe al tipo de vegetación típica de bosque montano; en general, las especies arbóreas que se comportan como hospederos de las orquídeas son Ocotea sp (Lauraceae), Podocarpus oleifolius D. Don (Podocarpaceae), Miconia spp (Melastomataceae), Eschweilera baguensis S. Mori (Lecythidaceae), Drymis granadensis L. f. (Winteraceae), Weinmania sp (Cunnoniaceae) (Tabla 4).

\section{Relaciones Fitogeográficas}

El número de especies reportadas para el Santuario Histórico de Machu Picchu (SHMP) es de 421 en 89 géneros (Núñez, 1992; Nauray, 2000; Galiano et al., 2001). Para el bosque nublado de Pillahuata se reportan 179 especies en 57 géneros. Para la reserva de biosfera del Manu (Galiano, 2000) es de 496 especies en 100 géneros. Para el presente estudio se reportan 206 especies en 58 géneros. La zona con mayor número de géneros y

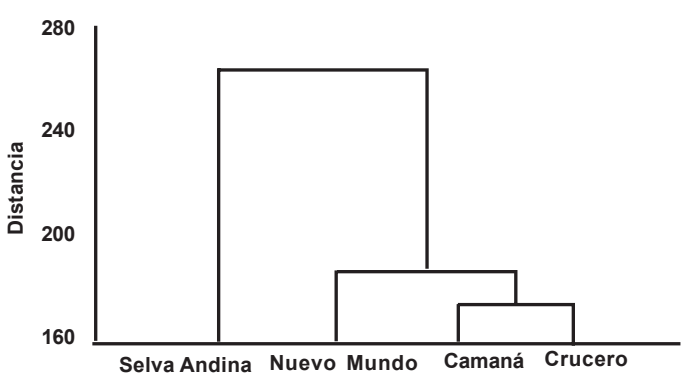

Figura 1. Dendrograma de Distancia Euclideana para las cuatro localidades en estudio.

especies es el SHMP en relación con el BNP, PNM y zona de estudio; sin embargo, respecto a ésta habría que tener en cuenta que es el primer y único estudio (hasta el momento) a detalle de la familia Orchidaceae, lo cual no se dio en el SHMP donde son varios los estudios realizados. Podríamos afirmar que llevando a cabo más estudios en la provincia de San Ignacio (Cajamarca), el número de reportes aumentaría considerablemente.

Según los estudios en el norte del Perú: Young y León (1999), Foster y Beltrán (1997) y el presente trabajo en comparación con las evaluaciones realizadas en el sur de Perú: Núñez (1992), Nauray (2000) y Galiano et al. (2001); SHMP: Moscoso (1995); BNP: Galiano et al. (2002); en el Manu se hallan 87 géneros comunes, los cuales tendrían un am-

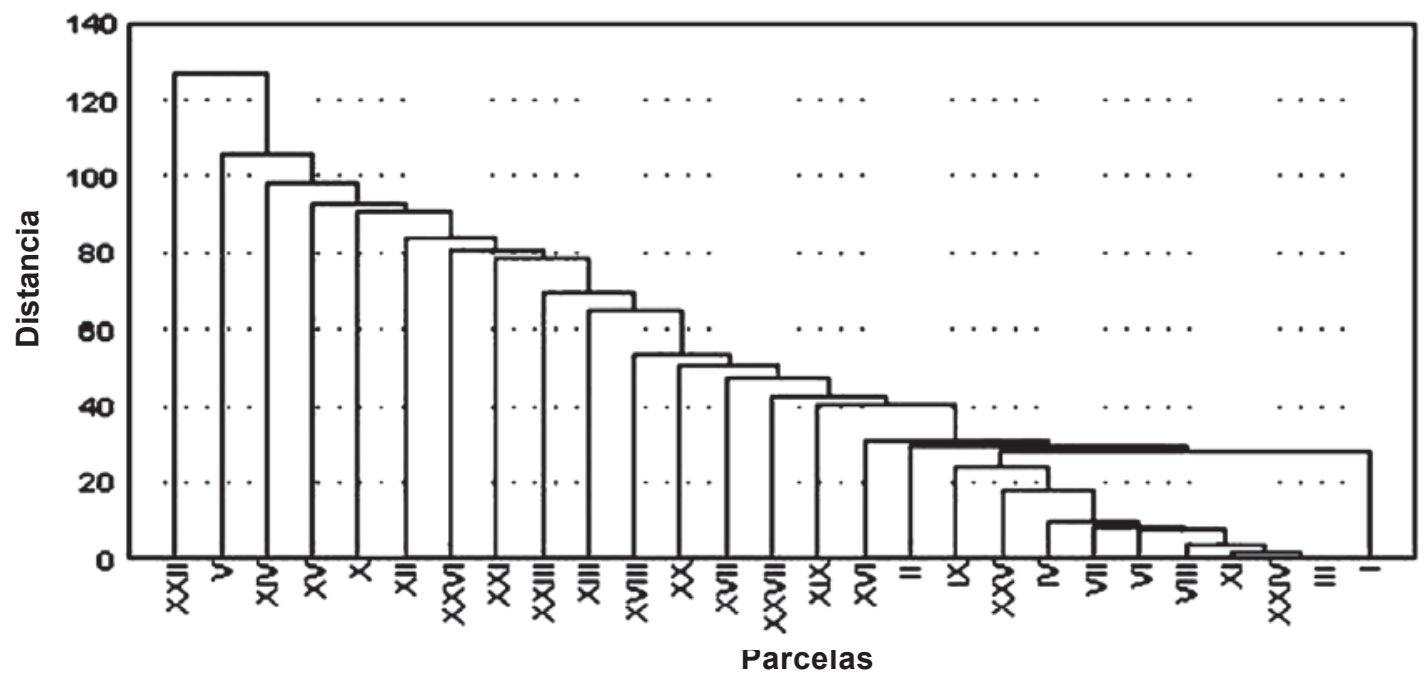

Figura 2. Dendrograma para los 27 cuadrantes en las localidades de estudio, provincia San Ignacio (Cajamarca). 
Tabla 4. Número de especies en los diferentes tipos de hábitos.

\begin{tabular}{lc}
\hline Hábito & No. Especies \\
\hline Epífito & 171 \\
Terrrestre & 43 \\
Litófito & 2 \\
\hline
\end{tabular}

plio rango de distribución geográfica y altitudinal (Apéndice 1).

Los géneros reportados sólo para evaluaciones realizadas en el sur de Perú (SHMP, BNP y PNM) son en número de 45; mientras los géneros reportados sólo para evaluaciones realizadas en el norte de Perú son 24 (Apéndice 2).

La alta diversidad de orquídeas reportada para el área de estudio confirma los datos mencionados por Gentry (1992), que las zonas con mayor diversidad son aquellas más cercanas a la base de las montañas entre los 1500 a $2500 \mathrm{~m}$. Un aspecto importante para esta alta diversidad es la zona de vida en la cual está ubicada el área de estudio y el buen estado de conservación del bosque, a pesar de que la extracción de especies maderables y la apertura de áreas para la agricultura y ganadería se hayan dado mayormente en las zonas no escarpadas.

\section{Agradecimientos}

Al Jardín Botánico de Missouri por la beca auspiciada por la John and Mac Arthur Foundation. A las Becas Elizabeth E. Bascom para Mujeres Botánicas Latinoamericanas concedidas por el Missouri Botanical Garden. Al curador del Missouri Botanical Garden, Ing. Rodolfo Vásquez Martínez y a la bióloga Rocío del Pilar Rojas Gonzáles por su permanente asistencia. Al biólogo Alfredo Tupayachi H., biólogo José Campos de la Cruz, biólogo Wilfredo Chávez. A los especialistas mencionados en el presente artículo por sus identificaciones y colaboración desinteresada. Por el apoyo en la consulta del herbario agradezco al Departamento de Botánica del Herbario del Missouri Botanical Garden y del Museo de Historia Natural de la Universidad Nacional Mayor de San Marcos.

\section{Literatura citada}

Foster R. \& H. Beltrán. 1997. The Cordillera del Condor Region of Ecuador and Peru: A Biological assessment. Conservation International. Washington. EE.UU.

Franco L.J., et al. 1995. Manual de Ecología, tercera edición. México, Ed. Trillas.

Galiano W., P. Núñez, A. Monteagudo, L. Valenzuela, 2000. Las Orquídeas y Plantas Ornamentales de la Reserva de la Biosfera del Manu. UniónComunidad Europea y la UNSAAC. Lima

Gentry A. 1992. Diversity and floristic Composition of Andean forest of Peru And Adjacent countries Implication for their conservation. Memorias del Museo de Historia Natural, UNMSM (Lima) 21:11-29.

Krebs C. 1989. Ecological Metodology, segunda edición. New York: University of British Columbia.

Mabberley D.J. 1997. The Plant Book, segunda edición. EEUU. Cambridge University.

Matteuci S.D. \& A. Colma. 1982. Metodología para el estudio de la vegetación. Washington DC. Secretaría General de la Organización de los Estados Americanos.

Moscoso D. 1995. Diversidad de la familia Orchidaceae en el Bosque Nublado de Pillahuata. Tesis para optar el título de Biólogo. Facultad de Ciencias Biológicas, UNSAAC, Cusco.

Nauray W. 2000. Diversidad, distribución, formas de vida y fenología floral de la familia Orchidaceae L. en el sector de Wiñay Wayna, Santuario Histórico de Machu Picchu. Tesis para optar el título de Biólogo. Facultad de Ciencias Biológicas, UNSAAC, Cusco.

Núñez P. 1992. Orchids in the Historic Sanctuary of Machu Picchu. Selbyana 13:159-160.

Palomino W. 1999. Diversidad y asociación arbórea en el Bosque Nublado de San Pedro, Reserva de Biosfera del Manu. Tesis para optar el título de Biólogo. Facultad de Ciencias Biológicas, UNSAAC, Cusco.

Vásquez R., R. Gonzales, C. Díaz y J. Campos, 1999. Caracterización cualitativa de la vegetación del Cerro Picorana, Cajamarca, Perú. Proyecto auspiciado por la John D. and Catherine T. Mac Arthur Foundation y la WWF-Programa Vacíos de Información Botánica. Jardín Botánico de Missouri.

Young K. \& B. Leon, 1999. «Peru's Humid Eastern montane Forest: An overview use and physical settings, biological diversity, human use and settlement, and conservation needs». DIVA, Technical Report 5. 


\section{Apéndice 1. Géneros comunes entre las evaluaciones al norte y sur de \\ Perú.}

Estudios en el sur de Perú: Núñez (1992), Nauray (2000) y Galiano et al. (2001); SHMP: Moscoso (1995); BNP: Galiano et al. (2002); Manu, en comparación con estudios en el norte del Perú: Estudios en el norte del Perú: Young y León (1999), Foster y Beltrán (1997), presente estudio San Ignacio (Cajamarca).

Altensteinia, Anguloa, Barbosella, Bletia, Brachystele, Brachionidium, Brassia, Bulbophyllum, Catasetum, Cleistes, Comparetia, Cranichis, Cryptocentrum, Cyclopogon, Cyrtochilum, Cirtopodium, Dichaea, Elleanthus, Epidendrum, Encyclia, Epylina, Epistephium, Erytrodes, Galeottia, Gongora, Gomphichis, Govenia, Habenaria, Hofmeisterella, Houlletia, Huntleya, Kefersteinia, Kreodanthus, Lepanthopsis, Lepanthes, Liparis, Lockhartia, Lueddemannia,Lycaste, Malaxis, Masdevallia,Maxillaria,Mormolyca, Mormodes, Myoxanthus, Neodryas, Octomeria, Odontoglossum, Oeceoclades, Oliveriana, Oncidium, Otoglossum,Pachyphyllum, Phragmipedium, Phragmipedium,Peristeria,Platystele, Pleurothallis, Polystachya,Ponthieva, Psilochilus,Prescottia,Psygmorchis, Pterichis, Rodriguezia, Rusbyella, Sauroglossum, Sarcoglottis, Scelochilus, Scaphyglottis, Sigmatostalix, Sobralia, Solennidiopsis, Stellilabium, Stenorrynchos, Stenia, Stenoptera, Stelis, Schomburgkia,Telipogon, Trichoceros.

\section{Apéndice 2. Géneros no comunes entre las evaluaciones al norte y sur del Perú.}

Estudios en el sur dePerú: Núñez(1992), Nauray (2000), Galiano et al. (2001); SHMP: Moscoso (1995); BNP: Galiano et al. (2000); Manu.

Aa,Apatostelis, Amblostoma, Batemania, Brachtia, Brassavola,Buchtienia, Rusbyella, Chloraea, Buesiella, Campylocentrum, Cattleya, Coccineorchis, Cochleantes, Crypthora, Cyrtidiorchis, Diadenium, Diotho nea, Eulophia, Frondaria,Ionopsis, Lycomormium, Macrademia, Macroclinium, Mesospinidium, Miltoniopsis, Neourbania, Nide ma, Myrosmodes, Ornitocephalus, Palmorchis, Paphinia, Pelexia, Plectrophora, Pithyphyllum,Sacoila, Spirantes, Stanhopea, Ttrigonidium, Trizeuxis, Vanilla,Xerorchis.

Estudios en el Norte del Perú: Young y León (1999), Foster y Beltrán (1997), presente estudio San Ignacio (Cajamarca)

Ackermani, Centropetalum, Corymborchis, Chrysocycnis, Cochlioda, Dresslerella, Eltropectis, Eriopsis, Eurystyles, Exalaria, Fernandezia, Galeandra,Hapalorchis, Lueddemannia, Miltonia, Notylia,Peristeria,Porroglossum, Restrepia, Restrepiopsis, Se rtifera, Susteloglossum, Trichocentrum, Trisetella. 